\title{
A simple and effective first optical image processing experiment
}

\section{Dale Olson}

Dale W. Olson, "A simple and effective first optical image processing experiment," Proc. SPIE 9665, Tenth International Topical Meeting on Education and Training in Optics and Photonics, 96651X (3 June 2007); doi: $10.1117 / 12.2211139$

SDIE Event: Tenth International Topical Meeting on Education and Training in Optics and Photonics, 2007, Ottawa, Ontario, Canada 


\title{
A simple and effective 'first' optical image processing experiment
}

\author{
Dale W. Olson, Physics Department, University of Northern Iowa, Cedar Falls, IA \\ 50614-0150
}

Optical image processing experiments can contribute to an understanding of optical diffraction and lens image formation. We are trying to discover a highly effective way of introducing lens imaging and related topics, light scattering, point sources, spatially coherent light, image processing, in a laboratory-based holography-centered introductory optics course serving a mixture of physics, chemistry, and science education sophomores and juniors. As an early experiment in this course, a microscope slide bearing opaque stick-on letters forming a word such as PAL is back-lighted by a point source of laser light. The surround for the letter $\mathrm{A}$ is transparent, while the surround for the letters $\mathrm{P}$ and $\mathrm{L}$ is made translucent with Scotch MAGIC ${ }^{\mathrm{TM}}$ tape. A 20-cm focal length converging lens forms a bright image of PAL on a screen, and also an image of the laser point source in a (transform) plane between the lens and the screen. Students are startled when they see that they can choose to pass only the image of the letter "A" or only the images of "P" and "L," by very simple manipulations in the transform plane. The interpretation of these experiments is challenging for some students, and the experiments can lead to a significant amount of discussion. Useful explanatory ray diagrams will be presented. Many demonstrations of optical image processing require long focal length lenses and precise manipulation of somewhat complex passing/blocking filters. In contrast these experiments are easy to set up and easy to perform. Students can fabricate the required objects in a matter of minutes. The use of zero-order laser light helps students discover the essential simplicity of the ideas underlying image processing. The simultaneous presence of both scattered (spatially incoherent) and not scattered (spatially coherent) laser light is thought provoking. Current explorations to further develop these and other closely-related experiments will also be described. 